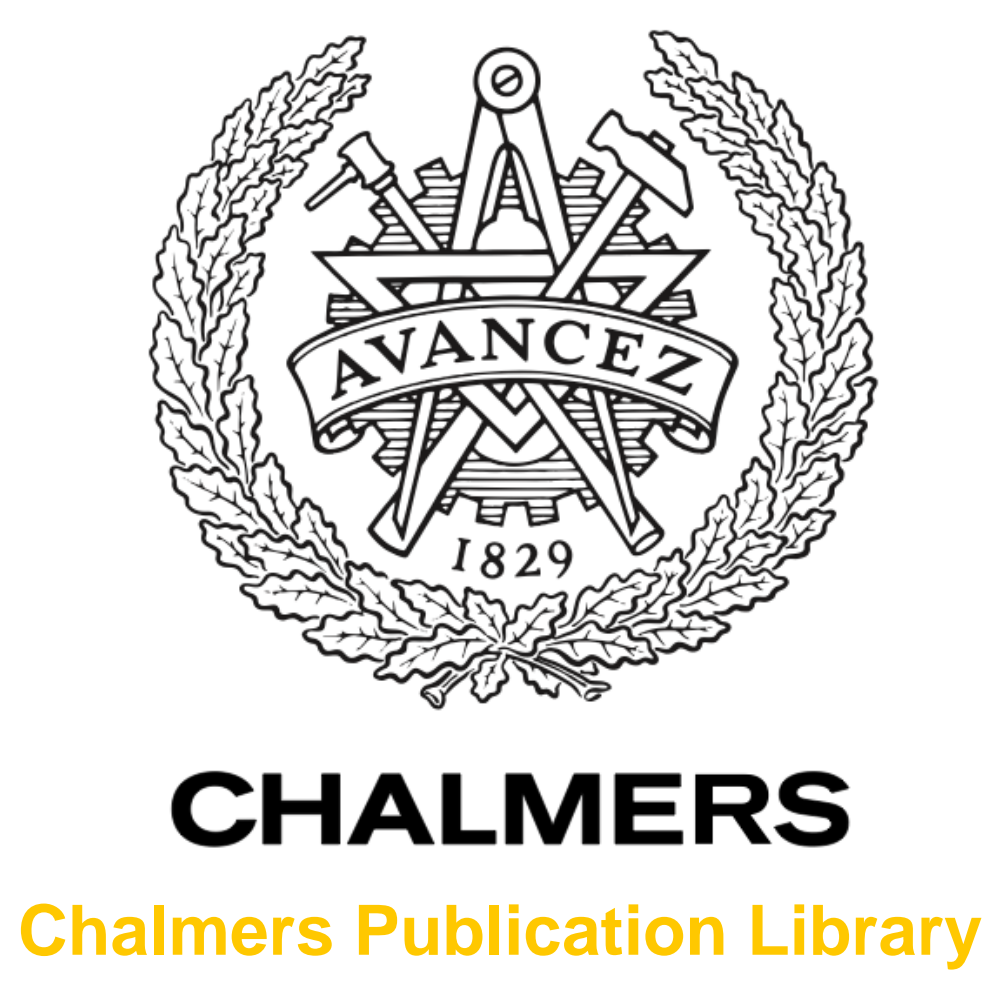

\title{
On Statistics of the Measured Antenna Efficiency in a Reverberation Chamber
}

This document has been downloaded from Chalmers Publication Library (CPL). It is the author's version of a work that was accepted for publication in:

IEEE Transactions on Antennas and Propagation (ISSN: 0018-926X)

Citation for the published paper:

Chen, X. (2013) "On Statistics of the Measured Antenna Efficiency in a Reverberation

Chamber". IEEE Transactions on Antennas and Propagation, vol. 61(11), pp. 5417-5427.

Downloaded from: http://publications.lib.chalmers.se/publication/186325

Notice: Changes introduced as a result of publishing processes such as copy-editing and formatting may not be reflected in this document. For a definitive version of this work, please refer to the published source. Please note that access to the published version might require a subscription. 


\title{
On Statistics of the Measured Antenna Efficiency in a Reverberation Chamber
}

\author{
Xiaoming Chen
}

\begin{abstract}
In this paper, the distribution of the measured antenna efficiency in a reverberation chamber (RC) is derived, based on which the statistics (e.g., mean and variance) of the measured antenna efficiency are studied. It is shown that the standard method (proposed in IEC 61000-4-21) for measuring antenna efficiency results in a consistent estimate. However, the standard estimator is only asymptotically unbiased (as the independent sample number $N$ goes to infinity) and it is biased for a finite $N$. The derived analytical expressions for the statistics of the antenna efficiency estimator are verified by simulations as well as RC measurements. In order to use the analytical expressions of the estimation statistics in practice, one needs to estimate the independent sample number $N$ from the measurement. This can be done based on a single measurement. Thus, using the derived estimation statistics, one can characterize the measurement performance of the antenna efficiency based on a single measurement, instead of directly estimating the statistics by performing many independent measurements.
\end{abstract}

Index Terms-Antenna efficiency, measurement, reverberation chamber (RC).

\section{INTRODUCTION}

$\mathbf{T}$ HE LAST decade has witnessed increasing popularity of using reverberation chambers (RCs) for measuring antenna efficiencies [1]; ergodic capacities of multiantenna systems [2], [3]; and active wireless devices [4], [6]. All of these applications necessitate a calibration of the $\mathrm{RC}$ by performing a reference measurement to determine the average power transfer function $P_{\text {ref }}$ [7]. Therefore, the measurement uncertainty of $P_{\text {ref }}$ affects measurement accuracies for all of these applications. The measurement uncertainty of $P_{\text {ref }}$ has been studied experimentally in [7] and [8]. However, to date, there are limited studies on the measurement uncertainties for those applications in [1]-[6]; and it might be dangerous to trust measurement results without knowing their measurement uncertainty.

The interest of this paper is on the statistics of the measured antenna efficiency in the RC, based on which the efficiency measurement uncertainty can be readily derived or estimated. Note that different methods of measuring antenna efficiency in an RC have been proposed to get rid of the reference measurement. For example, a time-reversal method (that is applicable for ultra wideband antennas only) was presented in [9]; a re-

Manuscript received May 12, 2013; revised July 02, 2013; accepted July 30, 2013. Date of publication August 06, 2013; date of current version October 28, 2013.

The author is with the Department of Signals and Systems, Chalmers University of Technology, Gothenburg 41296, Sweden (e-mail: xiaoming.chen@chalmers.se).

Color versions of one or more of the figures in this paper are available online at http://ieeexplore.ieee.org.

Digital Object Identifier 10.1109/TAP.2013.2276920 flection-based method (which may suffer poor accuracy in the return loss) was presented in [10]; by using two identical antennas under test, the antenna efficiency can be measured by estimating the quality factor of the RC [11]; several methods for measuring antenna efficiencies based on the estimation of the RC decay time were presented in [12], where an empirical study on the measurement uncertainty was presented. Nevertheless, since the reference measurement is necessary for all of the other applications and that the standard method (that involves a reference measurement) [1] is widely used (e.g., [13]), we prefer to study the statistics of the measured antenna efficiency by using the standard method [1]. It should be noted that the measurement uncertainty of the antenna efficiency has been preliminarily studied in terms of standard deviation (STD) in [14], where an approximation of the estimation variance is given. However, to date, the distribution of the measured antenna efficiency is unknown. Therefore, an exact expression of the estimation variance is missing; and other important estimation metrics, such as estimation bias and mean square error (MSE), have been overlooked in the literature.

A statistical RC measurement is incomplete without analysis of the measurement uncertainty. Although the standard method has been used ubiquitously [1], its statistics have not been studied thoroughly to date. Therefore, it is of fundamental importance to derive the distribution and the statistics of the standard antenna efficiency estimator. To that end, we analytically study the estimation statistics (e.g., bias, variance, and MSE) of the antenna efficiency estimate by first deriving the distribution of the standard efficiency estimator (i.e., the standard method) [1] in this paper. The derived statistics are verified using numerical simulations as well as RC measurements. Based on the statistics, we propose an unbiased efficiency estimator with improved estimation performance when the independent sample number is small. It should be noted that, in practice, the independent sample number has to be large for reliable estimation of antenna efficiency. Thus, the proposed unbiased efficiency estimator may be of little practical use, but it is theoretically a better estimator. It is also shown that by using the analytical expressions of the estimation statistics, the measurement performance of the antenna efficiency can be characterized based on a single measurement, instead of direct evaluation by performing many independent measurements. Furthermore, discussions on practical RC designs for more accurate antenna efficiency measurement are given in Section IV based on the statistics of the RC measurement.

\section{Statistics of Measured Antenna Efficiency}

The standard method for measuring the antenna efficiency in an RC is to first measure the average power transfer function $P_{\text {ref }}$ between a reference antenna (with known antenna ef- 
ficiency $\left.e_{\text {ref }}\right)$ and a fixed antenna; then measure that between the AUT and the fixed antenna $P_{\mathrm{AUT}}$. One can regard the fixed antenna as the transmitting antenna, and the reference antenna and the AUT as receiving antennas, respectively. During both measurements, the reference antenna and the AUT must be placed in the chamber in order to keep the same RC loading. Based on the measurements, the total radiation efficiency (that is simply referred to as antenna efficiency throughout this paper) of the AUT is estimated as

$$
\hat{e}_{\mathrm{AUT}}=\frac{\hat{P}_{\mathrm{AUT}}}{\frac{\hat{P}_{\mathrm{ref}}}{e_{\mathrm{ref}}}}
$$

where the hat denotes the estimate, for example, $\hat{e}_{\mathrm{AUT}}$ is the estimate of the antenna efficiency $e_{\mathrm{AUT}}$, and $\hat{P}_{\mathrm{AUT}}$ and $\hat{P}_{\text {ref }}$ are the estimates of the average power transfer functions received by the AUT $P_{\text {AUt }}$ and the reference antenna $P_{\text {ref }}$, respectively. Note that the antenna efficiency of the transmitting antenna is calibrated out by (1).

Denoting the net average power transfer functions as

$$
\begin{aligned}
\hat{G}_{\mathrm{AUT}} & =\frac{\hat{P}_{\mathrm{AUT}}}{e_{\mathrm{AUT}}} \\
\hat{G}_{\mathrm{ref}} & =\frac{\hat{P}_{\mathrm{ref}}}{e_{\mathrm{ref}}}
\end{aligned}
$$

respectively, (1) can be rewritten as

$$
\hat{e}_{\mathrm{AUT}}=\frac{e_{\mathrm{AUT}} \hat{G}_{\mathrm{AUT}}}{\hat{G}_{\mathrm{ref}}} .
$$

Since $e_{\mathrm{AUT}}$ is a constant, we are interested in the distribution of the random variable $\hat{G}_{\mathrm{AUT}} / \hat{G}_{\text {ref }}$ only. Assuming that there are $N$ independent random variables of the net power transfer functions

$$
\begin{aligned}
\hat{G}_{\mathrm{AUT}} & =\frac{1}{N} \sum_{i=1}^{N} G_{\mathrm{AUT}, i}, \\
\hat{G}_{\mathrm{ref}} & =\frac{1}{N} \sum_{i=1}^{N} G_{\mathrm{ref}, i}, \\
\frac{\hat{G}_{\mathrm{AUT}}}{\hat{G}_{\mathrm{ref}}} & =\frac{\sum_{i=1}^{N} G_{\mathrm{AUT}, i}}{\sum_{i=1}^{N} G_{\mathrm{ref}, i}} .
\end{aligned}
$$

Note that $E\left[\hat{G}_{\mathrm{AUT}}\right]=E\left[G_{\mathrm{AUT}, i}\right]=E\left[\hat{G}_{\mathrm{ref}}\right]=E\left[G_{\mathrm{ref}, i}\right]=$ $G_{0}$, where $E$ denotes the expectation. Since $G_{\mathrm{AUT}, i}$ and $G_{\mathrm{ref}, i}$ are independent and identically distributed (i.i.d.) random variables that are exponentially distributed [15], the numerator and the denominator in the right side of (5) follow Gamma distribution, Gamma $\left(N, G_{0}\right)$ [16]. The probability density function (pdf) of Gamma $\left(N, G_{0}\right)$ is

$$
f(x)=\frac{x^{N-1} \exp \left(\frac{-x}{G_{0}}\right)}{G_{0}^{N} \Gamma(N)}
$$

where $\Gamma$ is the Gamma function. Since $N$ is an integer, $\Gamma(N)=$ $(N-1)$ !, where ! represents the factorial operator. Note that this distribution approaches Gaussian as $N$ grows large due to the central limit theory.

For notational convenience, we denote $X=\sum_{i=1}^{N} G_{\mathrm{AUT}, i}$, $Y=\sum_{i=1}^{N} G_{\mathrm{ref}, i}$, and $Z=X / Y$. Hence, we are interested in the distribution of the random variable $Z$. For distinction, we denote the pdf of $Z$ as $f_{Z}$. Thus, (6) is denoted as $f_{X}$ hereafter.

In order to determine $f_{Z}$, we need an auxiliary equation $\mathrm{U}=$ $\mathrm{Y}$. Hence, the group of equations for the multivariate transformation is

$$
\left\{\begin{array}{l}
Z=g_{1}(X, Y)=\frac{X}{Y} \\
U=g_{2}(X, Y)=Y
\end{array}\right.
$$

whose inverse map is

$$
\left\{\begin{array}{l}
X=g_{1}^{-1}(Z, U)=Z U \\
Y=g_{2}^{-1}(Z, U)=U .
\end{array}\right.
$$

The Jacobian transform is

$$
J=\left|\begin{array}{ll}
\frac{\partial g_{1}^{-1}}{\partial z} & \frac{\partial g_{2}^{-1}}{\partial z} \\
\frac{\partial g_{1}^{-1}}{\partial u} & \frac{\partial g_{2}^{-1}}{\partial u}
\end{array}\right|=u .
$$

The joint pdf of $\mathrm{Z}$ and $\mathrm{U}$ is [16]

$$
f_{Z, U}(z, u)=f_{X, Y}\left(g_{1}^{-1}, g_{2}^{-1}\right)|J|=f_{X, Y}(z u, u) u \text {. }
$$

Since $\mathrm{X}$ and $\mathrm{Y}$ are i.i.d.

$$
f_{X, Y}(z u, u)=f_{X}(z u) f_{X}(u)=\frac{z^{N-1} u^{2 N-2} \exp \left(-\frac{z u+u}{G_{0}}\right)}{\left(G_{0}^{N} \Gamma(N)\right)^{2}} .
$$

Substitute (11) into (10)

$$
f_{Z, U}(z, u)=\frac{z^{N-1} u^{2 N-1} \exp \left(-(z u+u) / G_{0}\right)}{\left(G_{0}^{N} \Gamma(N)\right)^{2}} .
$$

In order to obtain $f_{Z}$, we integrate both sides of (12) over $u$

$$
\begin{aligned}
f_{Z}(z) & =\int_{0}^{\infty} f_{Z, U}(z, u) d u=\frac{z^{N-1} \int_{0}^{\infty} u^{2 N-1} \exp \left(-\frac{(z+1) u}{G_{0}}\right) d u}{\left(G_{0}^{N} \Gamma(N)\right)^{2}} \\
& =\frac{\Gamma(2 N)}{(\Gamma(N))^{2}} \frac{z^{N-1}}{(1+z)^{2 N}} .
\end{aligned}
$$

Once the distribution is known, one can readily calculate the mean and the variance (and other moments) of the random variable $\mathrm{Z}$

$$
\begin{aligned}
E[Z] & =\int_{0}^{\infty} z f_{Z}(z, u) d u=\frac{N}{N-1}, \\
\operatorname{Var}[Z] & =\int_{0}^{\infty}(z-E[Z])^{2} f_{Z}(z, u) d u \\
& =\frac{N(2 N-1)}{(N-2)(N-1)^{2}}
\end{aligned}
$$


where $\operatorname{Var}$ denotes the variance operator. Note that $Z$ is related to the measured antenna efficiency by $\hat{e}_{\mathrm{AUT}}=e_{\mathrm{AUT}} Z$. Therefore, the mean and the variance of $\hat{e}_{\mathrm{AUT}}$ are

$$
\begin{aligned}
E\left[\hat{e}_{\mathrm{AUT}}\right] & =\frac{N}{N-1} e_{\mathrm{AUT}} \\
\operatorname{Var}\left[\hat{e}_{\mathrm{AUT}}\right] & =\frac{N(2 N-1)}{(N-2)(N-1)^{2}} e_{\mathrm{AUT}}^{2}
\end{aligned}
$$

respectively. It can be seen from (15) that the efficiency estimator (1), that is, the standard method for measuring antenna efficiency in an RC, is asymptotically unbiased (as $N$ goes to infinity), and that its variance goes to zero as $N$ goes to infinity. Thus, the antenna efficiency estimator (1) is consistent [17]. Note that although the estimator (1) is asymptotically unbiased, it is biased for finite $N$. Nevertheless, it is straightforward to derive an unbiased estimator of the antenna efficiency based on (15)

$$
\hat{e}_{\mathrm{AUT}}^{\text {unbiased }}=\frac{N-1}{N} \frac{\hat{P}_{\mathrm{AUT}}}{\frac{\hat{P}_{\mathrm{ref}}}{e_{\mathrm{ref}}}} .
$$

The mean and the variance of the unbiased estimator (16) are

$$
\begin{aligned}
E\left[\hat{e}_{\mathrm{AUT}}^{\text {unbiased }}\right] & =e_{\mathrm{AUT}}, \\
\operatorname{Var}\left[\hat{e}_{\mathrm{AUT}}^{\text {unbiased }}\right] & =\frac{2 N-1}{N(N-2)} e_{\mathrm{AUT}}^{2}
\end{aligned}
$$

respectively.

The rmss of $\hat{e}_{\mathrm{AUT}}$ and $\hat{e}_{\mathrm{AUT}}^{\text {unbiased }}$ can be easily derived from (15) and (17), respectively

$$
\begin{aligned}
\operatorname{rms}\left[\hat{e}_{\mathrm{AUT}}\right] & =e_{\mathrm{AUT}} \sqrt{\frac{N(N+1)}{(N-2)(N-1)},} \\
\operatorname{rms}\left[\hat{e}_{\mathrm{AUT}}^{\text {unbiased }}\right] & =e_{\mathrm{AUT}} \sqrt{\frac{N^{2}-1}{N(N-2)} .}
\end{aligned}
$$

Note that in a preliminary study of the measurement uncertainty of $\hat{e}_{\mathrm{AUT}}[14]$, an approximation of $\operatorname{Var}\left[\hat{e}_{\mathrm{AUT}}\right]$ was given as

$$
\operatorname{Var}\left[\hat{e}_{\mathrm{AUT}}\right] \approx \frac{2}{N} e_{\mathrm{AUT}}^{2}
$$

Comparing (15) and (20), it can be seen that the approximation (20) is accurate for large $N$. The derivation of (20) in [14] is based on the first-order (2-D) Taylor expansion of the function $g_{1}(X, Y)$ (see (7)) in the neighborhood of $(E[X], E[Y])$. Elegant as it is, it fails to show the accuracy dependence on $N$. In order to show the $N$-dependence in the approximation (20) explicitly, an alternative derivation (that enables better physical interpretation of the approximation) is given in the Appendix.

As expected, the statistics of $\hat{e}_{\mathrm{AUT}}$ and $\hat{e}_{\mathrm{AUT}}^{\text {unbiased }}$, that is, (15) and (17)-(19) are functions of the independent sample number $N$, from which it can be seen that estimators (1) and (16) converge to $e_{\mathrm{AUT}}$ with a convergence rate of about $\sqrt{1 / N}$. Hence, it is important to have many independent samples for an accurate measurement of the antenna efficiency.
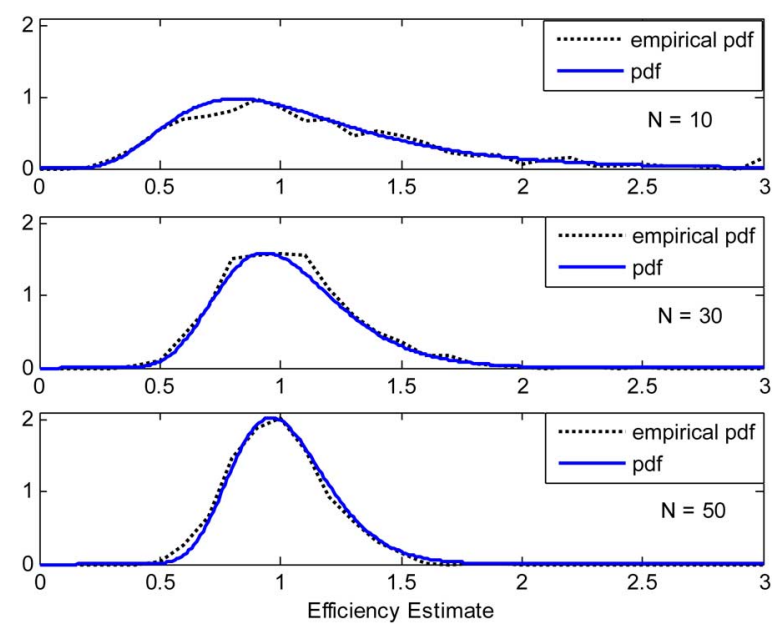

Fig. 1. Comparisons of the analytical pdf (13) and the corresponding empirical pdf for $N=10,30$, and 50 , respectively.

Since (1) is a biased estimator, a suitable performance metric of it is the MSE, that is, $\left.E\left[\hat{e}_{\mathrm{AUT}}-e_{\mathrm{AUT}}\right)^{2}\right]$, which can be easily derived from (15) as

$$
\operatorname{mse}\left[\hat{e}_{\mathrm{AUT}}\right]=\frac{2(N+1)}{(N-2)(N-1)} e_{\mathrm{AUT}}^{2} .
$$

Since (16) is unbiased, its MSE is equal to its variance (see (17)).

Note that in practice, the measured samples in an RC may be correlated, and that one needs to estimate the independent sample number $N$ from the measurements in order to evaluate the estimation performances of (1) and (16). Different estimators of the independent sample number can be found in the literature, for example, [8] and [20]-[23]. The choice of the $N$ estimator depends on the particular mode-stirring mechanisms employed in the RC, which is discussed in Section IV.

\section{Simulations}

In this section, we resort to simulations for verifying the derived statistics of the efficiency estimator. For simplicity and without loss of generality, we assume the antenna efficiency is unity (i.e., $e_{\mathrm{AUT}}=1$ ) throughout this section.

For each independent sample number $N$, we numerically generate 1000 samples (that follow i.i.d. exponential distribution) for $\hat{G}_{\text {AUT }}$ and $\hat{G}_{\text {ref }}$. As a result, we have 1000 realizations of $\hat{e} q_{\mathrm{AUT}}$ for each $N$, based on which we can obtain the empirical pdf, mean, variance, as well as other moment-based statistics.

Fig. 1 shows the comparison between the empirical pdf and the analytical pdf (13) for $N=10,30$, and 50, respectively. The good agreement between the analytical pdf and the empirical pdf verifies the derived pdf. Note that the first and second terms in the right-hand side of (13) result in extremely large and extremely small (non-negative) values, respectively. Therefore, it is numerically infeasible to use (13) for calculating the pdf for larger $N$. Fortunately, the moment-based statistics do not have such a constraint. 

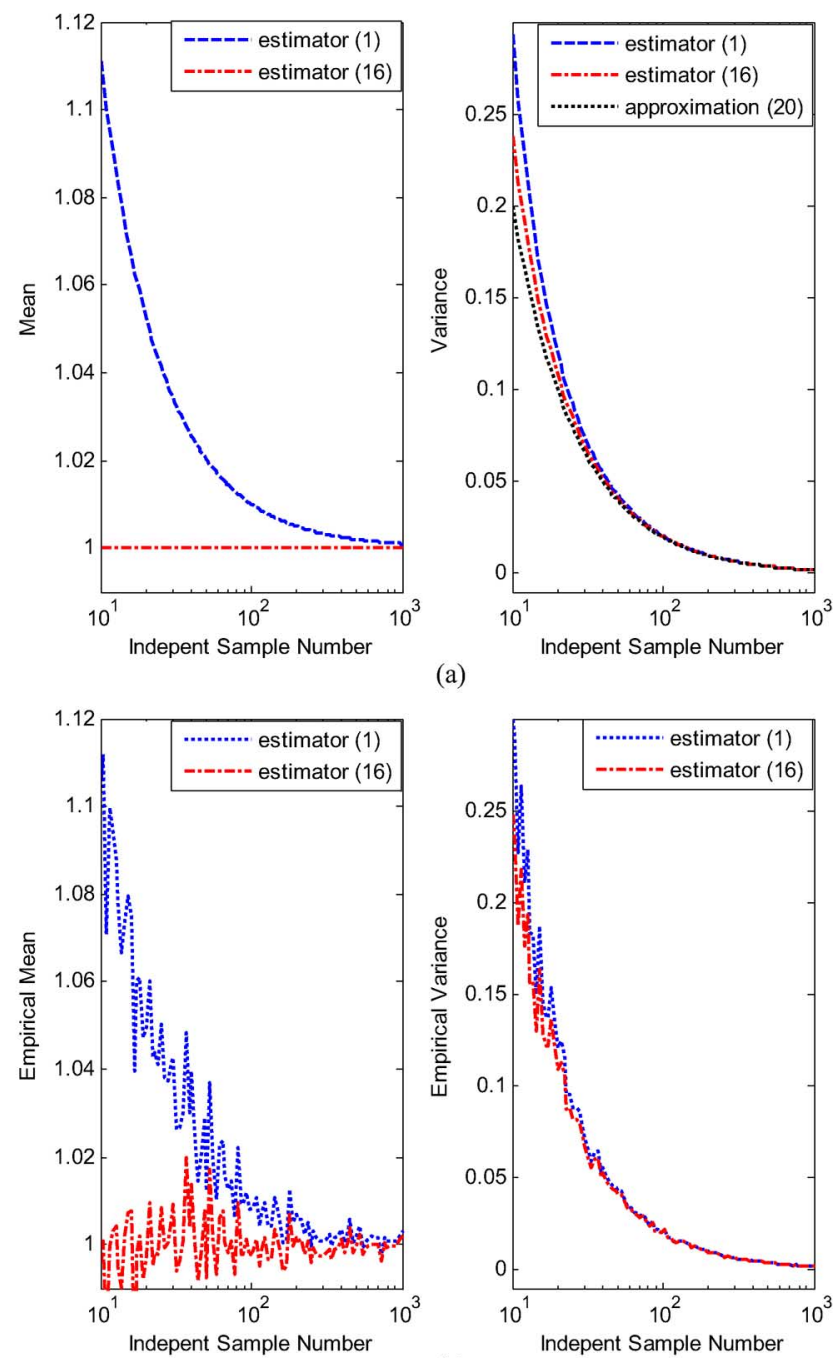

(a)

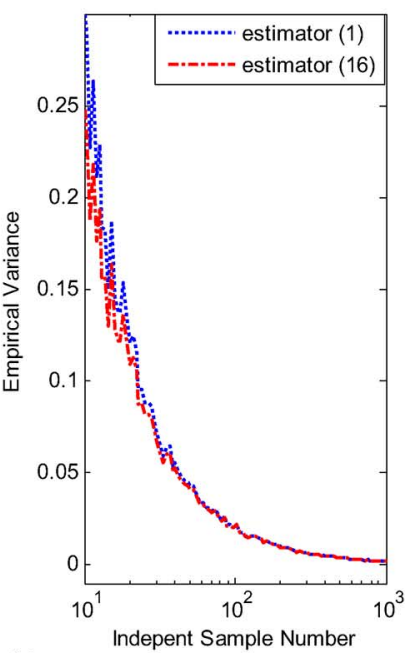

(b)

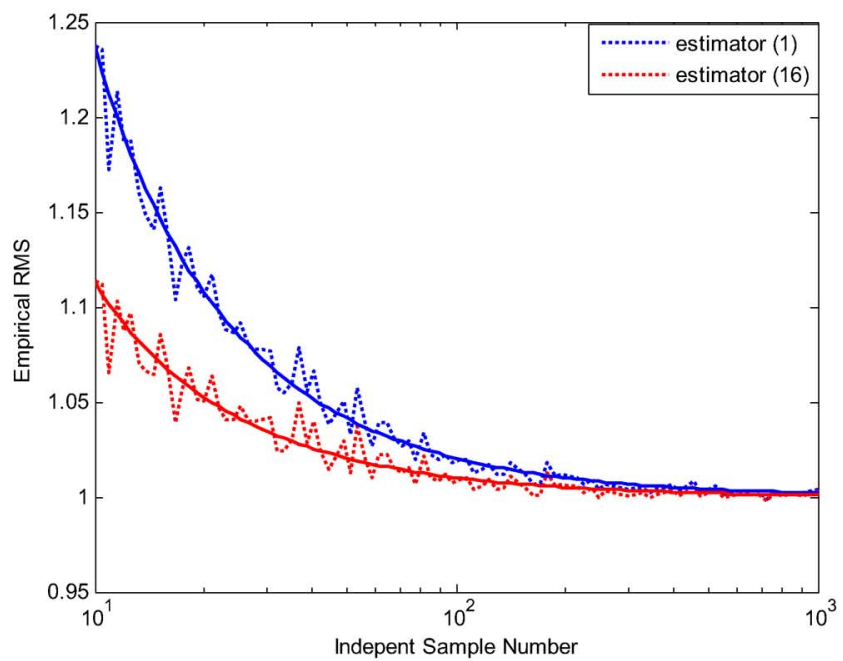

(c)

Fig. 2. Means and variances of estimators (1) and (16) for the case $e_{\mathrm{AUT}}=$ 1: (a) analytical mean and variance, where the variance approximation (20) is plotted for comparison; (b) empirical mean and variance; (c) analytical (solid) and empirical (dotted) rmss

Fig. 2 shows the analytical and empirical means, variances, and rmss of estimators (1) and (16) as a function of $N$, respec-

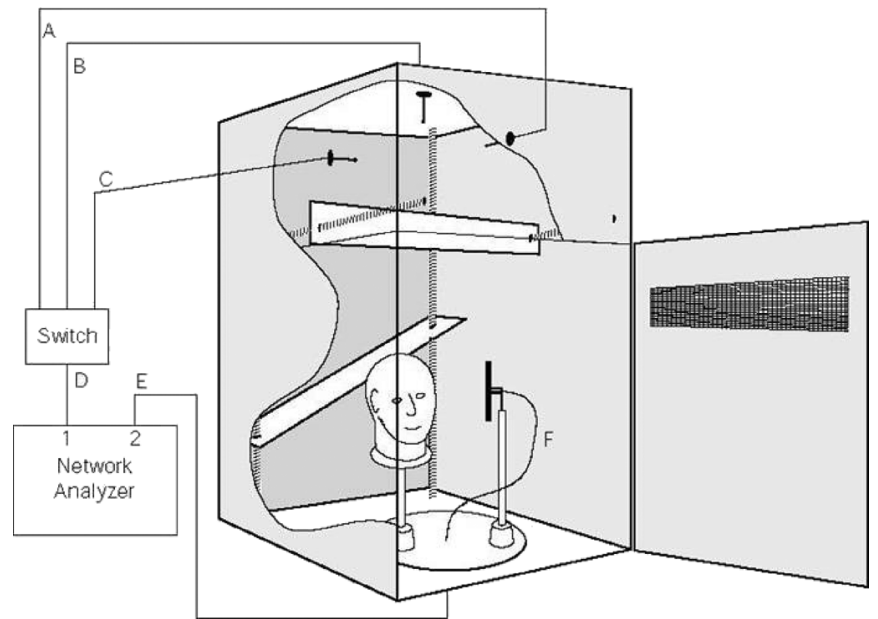

Fig. 3. Drawing of the RC with two mechanical plate stirrers, a platform, three wall antennas, and a head phantom.

tively. As can be seen, the analytical and empirical mean and variance agree with each other. It can also be seen that the unbiased estimator (16) offers (slight) improvement over the standard one (1) not only in estimation bias but also in estimation variance for small $N$. Nevertheless, the performances of the two estimators are indistinguishable for large $N$. Note that the approximation of the variance of $\hat{e}_{\mathrm{AUT}}$ (20) is also plotted in the right graph of Fig. 2(a) for comparison. It can be seen that this approximation serves as a lower bound that is tight for $N>30$. Thus, it can be used for uncertainty characterizations in practice (cf. [14]). Nevertheless, the variance expressions in (15) and (17) are as easy to calculate as the approximation (20); since they are the exact variance expression for $\hat{e}_{\mathrm{AUT}}$ and $\hat{e}_{\mathrm{AUT}}^{\text {unbiased }}$, respectively, we prefer to use (15) and (17) instead.

\section{MEASUREMENTS}

In order to further verify the derived statistics of the antenna efficiency estimators, extensive measurements were performed from 700 to $3000 \mathrm{MHz}$ (covering the most interesting telecommunication bands) in the Bluetest HP RC [7]. The RC used has a size of $1.80 \times 1.75 \times 1.25 \mathrm{~m}^{3}$ (a drawing of which is shown in Fig. 3). It has two mode-stirring plates, a turntable platform (on which a wideband discone antenna, used as the reference antenna, is mounted), and three half-bow-tie antennas mounted on three orthogonal walls (referred to as wall antennas hereafter). During the measurement, the turntable platform was moved stepwise to 20 platform-stirring positions (evenly distributed over one complete rotation); at each platform-stirring position, the two plates were moved simultaneously and stepwise to 50 positions (equally spanned over the total distances that they can travel along two walls inside the RC). At each stirrer position and for each wall antenna, a full frequency sweep was performed by a vector network analyzer (VNA) with a frequency step of $1 \mathrm{MHz}$, during which the scattering parameters (S-parameters) are sampled (as a function of frequency and stirring position). Hence, for each measurement, we have three wall antennas, 50 plate-stirring positions, and 20 platform-stirring positions. 
To facilitate the characterization of the antenna efficiency estimators, the same measurement sequence is repeated 12 times, each time with a different height/orientation of the reference antenna on the platform, that is, the reference antenna was placed at four different heights and at each height, it is placed with one vertical and two horizontal orientations (in radial and tangential directions of the platform), respectively. The heights and orientations are chosen to ensure independent measurements. In postprocessing, arbitrary pairs of antenna heights/orientations are chosen as the AUTs and the reference antennas, respectively, for estimating $e_{\mathrm{AUT}}$; and we introduce a $0-, 3-$, and 6-dB attenuator to the AUT (whose negative value in decibels is $e_{\mathrm{AUT}}$ ). Note that it is nontrivial to find the maximum number of independent samples of $\hat{e}_{\mathrm{AUT}}$ and $\hat{e}_{\mathrm{AUT}}^{\mathrm{unbiased}}$ from the 12 measurements. To be safe, we choose six pairs of distinct measurements to obtain six independent antenna efficiency estimates, which are used for evaluating the performance metric of the antenna efficiency estimator. For a good antenna design, $e_{\mathrm{AUT}}$ should probably be above $-1 \mathrm{~dB}$. However, in wireless communications, the antenna may be used in the presence of a human hand and head, for example, [18], where scenario $e_{\mathrm{AUT}}$ easily degrades to $-6 \mathrm{~dB}$ or even lower. In order to see the RC loading effect on the estimation performance of the antenna efficiency, the measurement procedure was repeated for two loading configurations: load 0 (unloaded RC) and load 1 (a head phantom that is equivalent to a human head in terms of microwave absorption). Hereafter, measured data from these two different loading configurations are simply referred to as load 0 and load 1 .

Since the standard method for measuring antenna efficiency results in a biased estimate (see (15)), the MSE is used as the performance metric for characterizing estimation bias and variance together. As shown in Section II, the MSE of $\hat{e}_{\mathrm{AUT}}$ can also be evaluated using the model (21), where the independent sample number $N$ can be estimated based on a reference measurement using the degrees of freedom (DoF) method [8]. For the sake of completeness, the DoF method is briefly presented below.

DoF Method: We divide the entire stirring sequence into three subsets and estimate the independent sample number for each subset while treating the other two subsets as observations. In this way, we avoid the potential problem of averaging frequency samples with different statistical properties. The three stirring sequences (from wall antennas, plates, and turntable platform) are independent in that they are from different stirring mechanisms, respectively. Thus, [19]

$$
N=N_{\text {ind,ant }} N_{\text {ind,pl }} N_{\text {ind,pf }}
$$

where each term at the right-hand side of (22) $N_{\text {ind,1 }}(l$ represents ant, $p l$, or $p f$ ) is estimated using the following procedure:

1) denote the complex (rectangular) field samples at the $m$ th antenna $(l=a n t)$, plate position $(l=p l)$, or platformstirring position $(l=p f)$ as a column vector $\mathbf{x}_{m}, m=$ $1 \ldots N_{1}$

2) concatenate $\mathbf{x}_{m}$ into a matrix $\mathbf{X}=\left[\mathbf{x}_{1} \ldots \mathbf{x}_{N_{l}}\right]$;

3) estimate the (unnormalized) correlation matrix of $\mathbf{X}$ as

$$
\mathbf{R}=\mathbf{X X}^{H}
$$
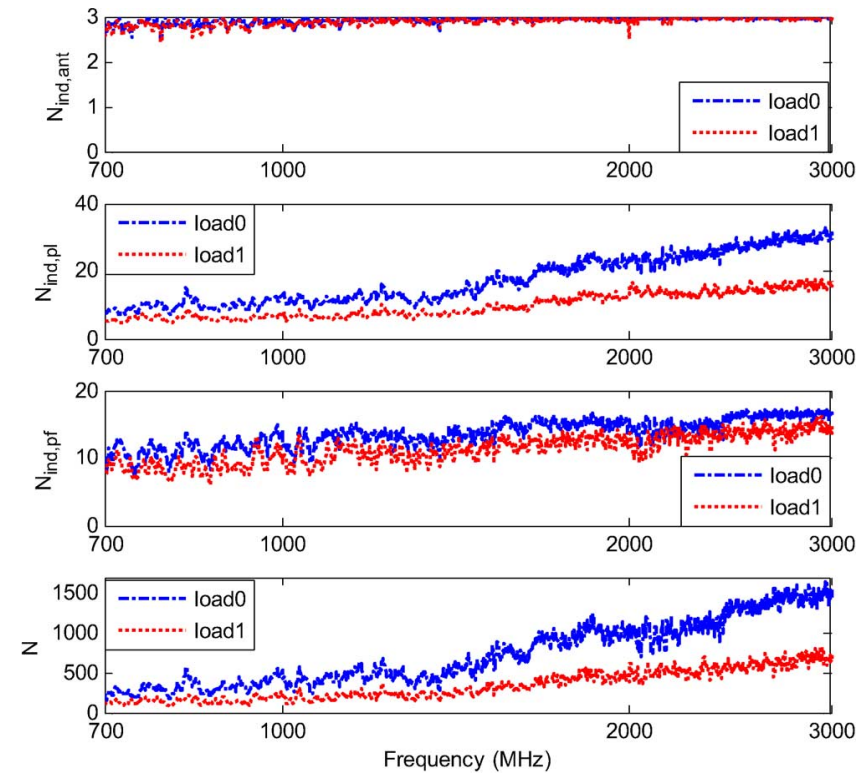

Fig. 4. Estimated independent sample numbers $N_{\text {ind,ant }}, N_{\text {ind, ant }}, N_{\text {ind,ant }}$, and $N$ using the DoF method.

4) the independent antenna $(l=a n t)$, mode-stirrer $(l=p l)$, or platform $(l=p f)$ sample number can be estimated as

$$
N_{\text {ind }, \mathrm{l}}=\frac{\operatorname{tr}(\mathbf{R})^{2}}{\operatorname{tr}\left(\mathbf{R}^{2}\right)}=\frac{\left(\sum_{i} \lambda_{i}\right)^{2}}{\sum_{i} \lambda_{i}^{2}}
$$

where $\mathbf{X}^{H}$ denotes the conjugate transpose of $\mathbf{X}$, tr represents the trace operator, and $\lambda_{i}$ represents the $i$ th eigenvalue of $\mathbf{R}$.

Fig. 4 shows the estimated independent sample numbers using the DoF method. It can be seen that the estimated $N$ increases with increasing frequency and decreases with increasing loading. Note that the DoF method inherently takes the RC loading (and, therefore, the unstirred component) effect into account. Also note that the DoF method is suitable for RCs with different (separable) stirring mechanisms. In case the stirring sequences generated by different stirring mechanisms are not separable, or there is only one stirring mechanism, one can either use the frequency-domain samples for estimating $N$ [20] (at the cost of frequency resolution and the risk of averaging samples with different statistical properties) or simply use other suitable $N$ estimators (for example, [21]-[23]).

Fig. 5 shows the empirical MSE of $\hat{e}_{\mathrm{AUT}}$ (for $e_{\mathrm{AUT}}=0$, -3 , and $-6 \mathrm{~dB}$ ) estimated based on independent measurements (solid) and the analytical MSE model (21) with $N$ estimated from a single measurement (dotted), respectively. Note that for clear exhibition, the empirical MSE is plotted using the following dB-transformation [7]:

$$
\mathrm{mse}_{\mathrm{dB}}=5 \log _{10} \frac{(1+\mathrm{mmse})}{(1-\mathrm{mse})}
$$

and that $40-\mathrm{MHz}$ frequency smoothing is applied to the empirical MSE curves before plotting. As can be seen, there are good 

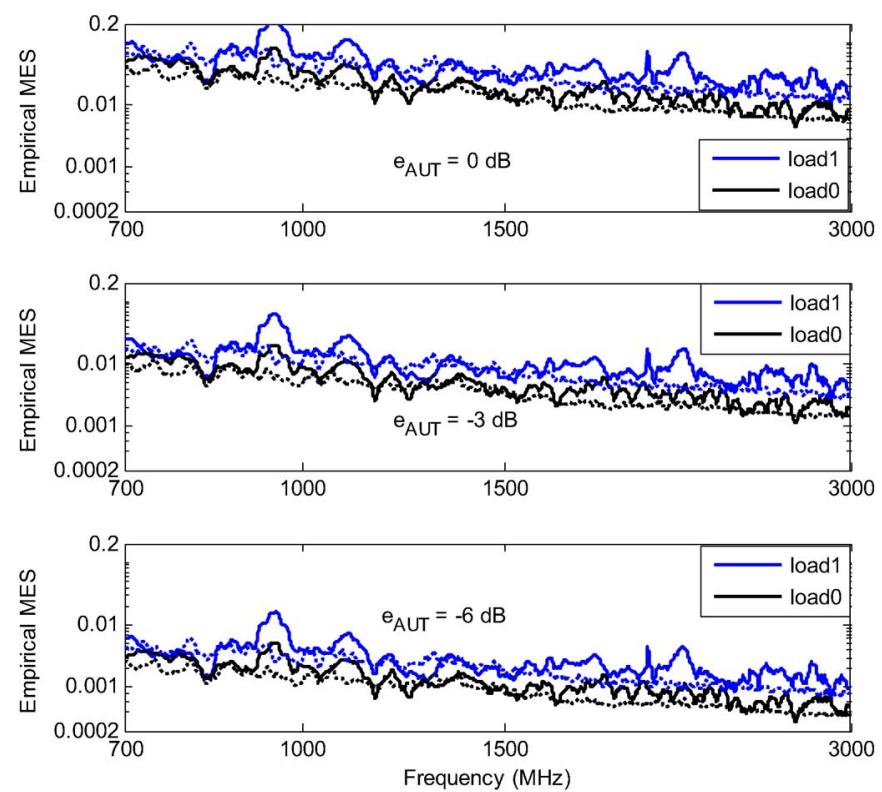

Fig. 5. Empirical MSE of $\hat{e}_{\mathrm{AUT}}$ based on independent measurements (solid) and the analytical MSE model (21) (dotted). The three graphs from top to bottom correspond to $e_{\mathrm{AUT}}=0,-3$, and $-6 \mathrm{~dB}$, respectively.

agreements between the MSE expressions (21) (with $N$ estimated from a single measurement) and the direct MSE estimate (based on the independent measurements). It can also be seen that the MSE of $\hat{e}_{\mathrm{AUT}}$ decreases with decreasing $e_{\mathrm{AUT}}$. This observation can be readily explained from the analytical MSE expression (21).

It can also be seen from (21) that the MSE decreases with increasing $N$ (with a convergence rate of about $1 / N$ ). From Fig. 4, it can be seen that the independent wall antenna number is equal to the number of wall antennas (i.e., three) from $1000 \mathrm{MHz}$ (and that the former is very close to the later from 700 to $1000 \mathrm{MHz}$ ). This implies that the independent wall antenna number can be readily increased by adding more wall antennas that are sufficiently away from each other (to avoid correlations). It can be seen from Fig. 4 that the independent plate and platform positions are strictly smaller than the numbers of plate and platform positions, respectively. This means that there will be no MSE improvement by increasing the number of plate and platform positions in the used RC. One can, of course, increase the radius of the turntable platform in order to increase the independent platform position number. However, the radius of the platform is limited by the dimensions of the RC (otherwise, the antenna under test will be too close to the walls). Possible ways to further improve the antenna efficiency measurement are, for example, to increase the size of the plates or optimize the shape of the plates. Nevertheless, these are out of the scope of this paper and, therefore, are left for future work.

Note that the estimation performances of $\hat{e}_{\mathrm{AUT}}$ and $\hat{e}_{\mathrm{AUT}}^{\text {unbiased }}$ are similar for large $N$. Therefore, for the sake of conciseness of this paper, the MSE of $\hat{e}_{\mathrm{AUT}}^{\text {unbiased }}$ (estimated using all stirring samples) is not shown here. In order to show the advantage of the proposed unbiased estimator (16) over the standard estimator (1), we re-evaluate the MSEs of $\hat{e}_{\mathrm{AUT}}$ and $\hat{e}_{\mathrm{AUT}}^{\text {unbiased }}$ for $N=15$ and 30. It can be seen from Fig. 4 that samples
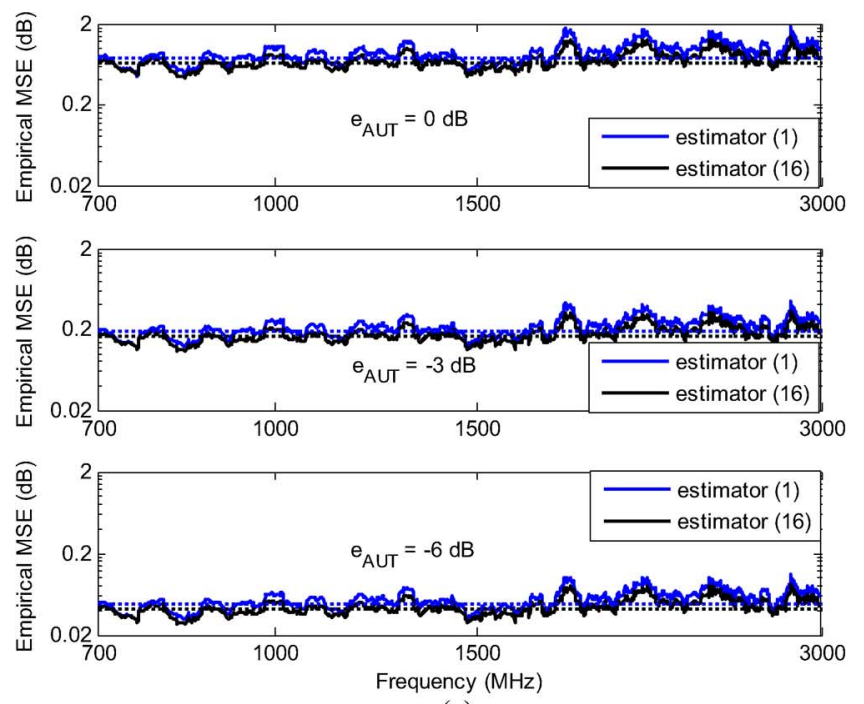

(a)
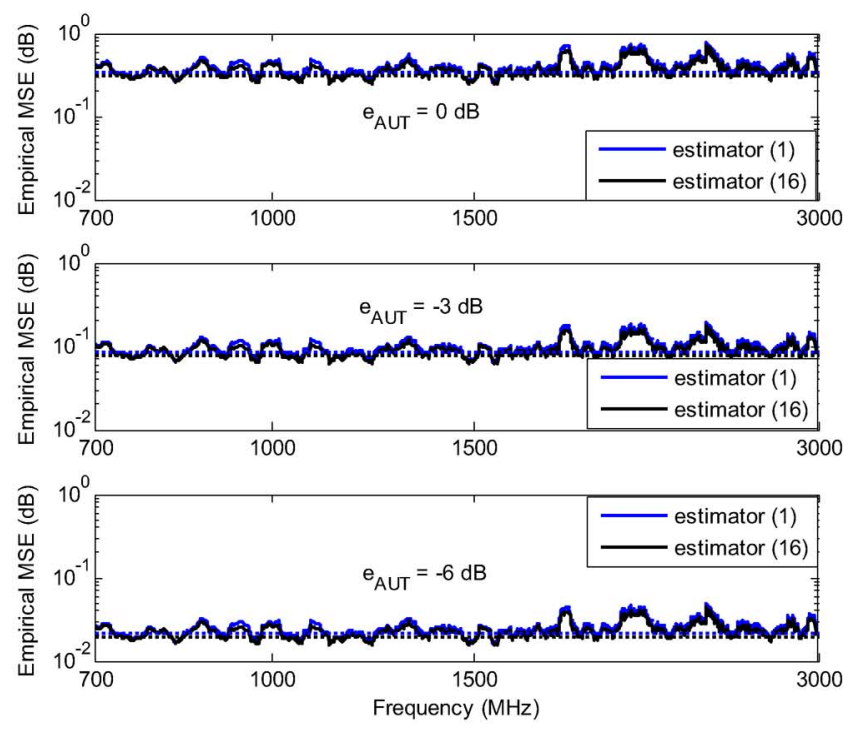

(b)

Fig. 6. Empirical MSEs of $\hat{e}_{\mathrm{AUT}}(1)$ and $\hat{e}_{\mathrm{AUT}}^{\mathrm{unbiased}}(16)$ based on independent measurements (solid) and the analytical MSE expressions (dotted) for (a) $N=$ 15 and (b) $N=30$ under the loading condition of load 0 .

from the three wall antennas are independent (in that the three wall antennas are orthogonally polarized with sufficient separations between them) and that the independent platform position number for either loading condition is about 10 at $700 \mathrm{MHz}$ (and increases with increasing frequency). Therefore, we choose the subsets of 5 or 10 platform positions (that are equally spaced), one plate position, and all three wall antennas, correspond to $N=15$ or 30 , for evaluating MSEs of $\hat{e}_{\mathrm{AUT}}$ and $\hat{e}_{\mathrm{AUT}}^{\text {unbiased }}$.

Fig. 6 shows the empirical MSEs of $\hat{e}_{\mathrm{AUT}}$ and $\hat{e}_{\mathrm{AUT}}^{\mathrm{unbiased}}$, estimated based on independent measurements and analytical MSE expressions (21) and (17) for $N=15$ and 30, respectively. Note that the MSE of $\hat{e}_{\mathrm{AUT}}^{\mathrm{unbiased}}$ is equal to its variance. Also note that $N=15$ and 30 are the independent sample numbers for load 0 and load 1. Thus, only the case of load 0 is shown in Fig. 6. As can be seen, there is small yet noticeable improvement of $\hat{e}_{\mathrm{AUT}}^{\text {unbiased }}$ over $\hat{e}_{\mathrm{AUT}}$ for $N=15$; nevertheless, the improvement becomes negligible for $N=30$. It should be noted that, in 
practice, the independent sample number should be much larger than 30 for a reliable antenna efficiency measurement. Thus, the standard estimator (1) is as good as the unbiased estimator (16) in a practical RC measurement, even though the latter is theoretically better (i.e., unbiased with slightly lower estimation variance for small $N$ values).

\section{CONCLUSION}

In this paper, we analytically derive the distribution of the antenna efficiency measured in an RC. Statistics of the antenna efficiency estimate are readily obtained using the derived distribution, from which it can be seen that the standard antenna efficiency estimator (1) is only asymptotically unbiased and that it results in an estimation bias when the number of independent sample numbers $N$ is small. As a result, an unbiased antenna efficiency estimator is proposed. The statistics of the unbiased estimator are also given. The analytically derived statistics of both estimators are verified by simulations and $\mathrm{RC}$ measurements. The results show that the unbiased estimator (slightly) outperforms the standard one for $N<30$, which implies that the standard antenna efficiency estimator (1) is as good as the unbiased one (16), since $N$ is usually much larger than 30 in practical RC measurements. Note that it is time-consuming to directly estimate the MSE of the measured antenna efficiency by performing many independent measurements. However, using the analytical expressions of the derived statistics (e.g., estimation bias, variance, and MSE) with estimated $N$, the measurement performance can be characterized based on a single measurement.

\section{APPENDIX}

\section{DERIVATION OF THE VARIANCE APPROXIMATION}

Since $\hat{G}_{\text {AUT }}$ and $\hat{G}_{\text {ref }}$ in (3) are averaged over $N$ independent samples of i.i.d. exponentially distributed $G_{\mathrm{AUT}, i}$ and $G_{\mathrm{ref}, i}$, respectively, for large $N, \hat{G}_{\mathrm{AUT}}$ and $\hat{G}_{\text {ref }}$ can be approximated by

$$
\begin{aligned}
\hat{G}_{\mathrm{AUT}} & =G_{0}+\Delta_{\mathrm{ref}}, \\
\hat{G}_{\mathrm{ref}} & =G_{0}+\Delta_{\mathrm{AUT}}
\end{aligned}
$$

respectively, where $\Delta_{\mathrm{AUT}}$ and $\Delta_{\mathrm{ref}}$ are assumed to be i.i.d., zero mean Gaussian errors, respectively, and $E\left[\hat{G}_{\mathrm{AUT}}\right]=E\left[G_{\mathrm{AUT}, i}\right]=E\left[\hat{G}_{\mathrm{ref}}\right]=E\left[G_{\mathrm{ref}, i}\right]=G_{0}$. Therefore, (3) can be approximated by

$$
\hat{e}_{\mathrm{AUT}} \approx e_{\mathrm{AUT}} \frac{G_{0}+\Delta_{\mathrm{ref}}}{G_{0}+\Delta_{\mathrm{AUT}}}=e_{\mathrm{AUT}} \frac{1+\Delta_{\mathrm{AUT}} / G_{0}}{1+\Delta_{\mathrm{ref}} G_{0}} .
$$

Multiplying $\left(1-\Delta_{\text {ref }} / G_{0}\right)$ to both the denominator and numerator of (26), one obtains

$$
\approx e_{\mathrm{AUT}} \frac{1+\Delta_{\mathrm{AUT}} / G_{0}-\Delta_{\mathrm{ref}} / G_{0}-\left(\frac{\Delta_{\mathrm{AUT}}}{G_{0}}\right)\left(\frac{\Delta_{\mathrm{ref}}}{G_{0}}\right)}{1-\left(\frac{\Delta_{\mathrm{ref}}}{G_{0}}\right)^{2}} .
$$

Note that due to the averaging (4), the (relative) errors $\Delta_{\mathrm{AUT}} / G_{0}$ and $\Delta_{\text {ref }} / G_{0}$ are small. (Actually, they approach zero as $N$ goes to infinity.) Hence, the second-order errors
$\left(\Delta_{\text {ref }} / G_{0}\right)^{2}$ and $\left(\Delta_{\text {AUT }} / G_{0}\right)\left(\Delta_{\text {ref }} / G_{0}\right)$ in (28) are negligible. Therefore, (24) can be further approximated by

$$
\hat{e}_{\mathrm{AUT}} \approx e_{\mathrm{AUT}}\left(1+\Delta_{\mathrm{AUT}} / G_{0}-\Delta_{\mathrm{ref}} / G_{0}\right) .
$$

As a result, the variance of $\hat{e}_{\mathrm{AUT}}$ can be approximated by

$$
\operatorname{var}\left[\hat{e}_{\mathrm{AUT}}\right] \approx\left(\frac{e_{\mathrm{AUT}}}{G_{0}}\right)^{2} \operatorname{var}\left[\Delta_{\mathrm{AUT}}-\Delta_{\mathrm{ref}}\right] .
$$

Since the reference measurement and the AUT measurement are two independent measurements, their errors $\Delta_{\mathrm{AUT}}$ and $\Delta_{\text {ref }}$ are independent. Thus, (30) can be rewritten as

$$
\begin{aligned}
\operatorname{var}\left[\hat{e}_{\mathrm{AUT}}\right] & \approx\left(\frac{e_{\mathrm{AUT}}}{G_{0}}\right)^{2}\left(\operatorname{var}\left[\Delta_{\mathrm{AUT}}\right]+\operatorname{var}[\Delta \mathrm{ref}]\right)^{2} \\
& =\left(\frac{e_{\mathrm{AUT}}}{G_{0}}\right)^{2}\left(\operatorname{var}\left[\hat{G}_{\mathrm{AUT}}\right]+\operatorname{var}\left[\hat{G}_{\mathrm{ref}}\right]\right)^{2} .
\end{aligned}
$$

The equality in (31) follows from (26) and the fact that $G_{0}$ is a constant. Duo to the averaging (4) and the fact that the variance of the i.i.d. exponentially distributed $G_{\mathrm{AUT}, i}$ and $G_{\mathrm{ref}, i}$ are $G_{0}^{2}$

$$
\operatorname{var}\left[\hat{G}_{\mathrm{AUT}}\right]=\operatorname{var}\left[\hat{G}_{\mathrm{ref}}\right]=\frac{G_{0}^{2}}{N} .
$$

Substituting (32) into (31), one obtains

$$
\operatorname{var}\left[\hat{e}_{\mathrm{AUT}}\right] \approx \frac{2 e_{\mathrm{AUT}}^{2}}{N} \text {. }
$$

\section{REFERENCES}

[1] IEC 61000-4-21: EMC 4: Testing and Measurement Techniques: Section 21: Reverberation Chamber Test Methods, , Int. Electrotech Comm, Geneva, Switzerland, 2001, Committee Draft.

[2] J. F. Valdes, M. A. Fernandez, A. M Gonzalez, and D. A. Hernandez, "The influence of efficiency on receive diversity and MIMO capacity for Rayleigh-fading channels," IEEE Trans. Antennas Propag., vol. 56, no. 5, pp. 1444-1450, May 2008.

[3] X. Chen, P.-S. Kildal, J. Carlsson, and J. Yang, "MRC diversity and MIMO capacity evaluations of multi-port antennas using reverberation chamber and anechoic chamber," IEEE Trans. Antennas Propag., vol. 61, no. 2, pp. 917-926, Feb. 2013.

[4] E. Genender, C. L. Holloway, K. A. Remley, J. M. Ladbury, G. Koepke, and H. Garbe, "Simulating the multipath channel with a reverberation chamber: Application to bit error rate measurements," IEEE Trans. Electromagn. Compat., vol. 52, no. 4, pp. 766-777, Nov. 2010.

[5] A. Sorrentino, G. Ferrara, and M. Migliaccio, "The reverberating chamber as a line-of-sight wireless channel emulator," IEEE Trans. Antennas Propag., vol. 56, no. 6, pp. 1825-1830, Jun. 2008.

[6] G. Ferrara, M. Migliaccio, and A. Sorrentino, "Characterization of GSM non-line-of-sight propagation channels generated in a reverberating chamber by using bit error rates," IEEE Trans. Electromagn. Compat., vol. 49, no. 3, pp. 467-473, Aug. 2007.

[7] P.-S. Kildal, X. Chen, C. Orlenius, M. Franzén, and C. Lötbäck Patané, "Characterization of reverberation chambers for OTA measurements of wireless devices: Physical formulations of channel matrix and new uncertainty formula," IEEE Trans. Antennas Propag., vol. 60, no. 8, pp. 3875-3891, Aug. 2012.

[8] X. Chen, "Experimental investigation of the number of independent samples and the measurement uncertainty in a reverberation chamber," IEEE Trans. Electromagn. Compat., to be published.

[9] G. L. Fur, P. Besnier, and A. Sharaiha, "Time reversal efficiency measurement in reverberation chamber," IEEE Trans. Antennas Propag., vol. 60, no. 6, pp. 2921-2928, Jun. 2012.

[10] P. Hallbjörner, "Reflective antenna efficiency measurements in reverberation chambers," Microw. Opt. Technol. Lett., vol. 30, no. 5, pp. 332-335, 2001. 
[11] H. G. Krauthäuser and M. Herbrig, "Yet another antenna efficiency measurement method in reverberation chambers," in Proc. IEEE Int. Symp. Electromagn. Compat., Jul. 25-30, 2010, pp. 536-540.

[12] C. L. Holloway, H. Shah, R. J. Pirkl, W. Young, J. Ladbury, and D. A. Hill, "Reverberation chamber techniques for determining the radiation and total efficiency of antennas," IEEE Trans. Antennas Propag., vol. 60, no. 4, pp. 1758-1770, Apr. 2012.

[13] S. J. Boyes, Y. Huang, N. Khiabani, P. J. Soh, and G. A. E. Vandenbosch, "Repeatability and uncertainty evaluations of on-body textile antenna efficiency measurements in a reverberation chamber," in Proc. Loughborough Antennas Propag. Conf., Loughborough, U.K., Nov. 12-13, 2012, pp. 1-5.

[14] X. Chen, "Measurement uncertainty of antenna efficiency in a reverberation chamber," IEEE Trans. Electromagn. Compat., to be published.

[15] J. G. Kostas and B. Boverie, "Statistical model for a mode-stirred chamber," IEEE Trans. Electromagn. Compat., vol. 33, no. 4, pp. 366-370, Nov. 1991.

[16] G. Grimmett and D. Stirzaker, Probability and Random Processes, 3rd ed. New York: Oxford University Press, 2001.

[17] S. M. Kay, Fundamentals of Statistical Signal Processing: Vol. I Estimation Theory. Upper Saddle River, NJ, USA: Prentice-Hall, 1993.

[18] V. Plicanic, B. K. Lau, A. Derneryd, and Z. Ying, "Actual diversity performance of a multiband diversity antenna with hand and head effects," IEEE Trans. Antennas Propag., vol. 57, no. 5, pp. 1547-1556, May 2009.

[19] A. Sorrentino, P.-S. Kildal, U. Carlberg, and E. Pucci, “Accuracy in reverberation chamber for wireless testing: Simple formulas for the number of independent samples," in Proc. 3rd Eur. Conf. Antennas Propag., Berlin, Germany, Mar. 2009, pp. 2673-2677.

[20] R. J. Pirkl, K. A. Remley, and C. S. L. Patané, "Reverberation chamber measurement correlation," IEEE Trans. Electromagn. Compat., vol. 54, no. 3, pp. 533-544, Jun. 2012.
[21] C. Lemoine, P. Besnier, and M. Drissi, "Estimating the effective sample size to select independent measurements in a reverberation chamber," IEEE Trans. Electromagn. Compat., vol. 50, no. 2, pp. 227-236, May 2008.

[22] G. Gradoni, V. Mariani Primiani, and F. Moglie, "Reverberation chamber as a multivariate process: FDTD evaluation of correlation matrix and independent positions," Progr. Electromagn. Res., vol. 133, pp. 217-234, 2013.

[23] H. G. Krauthauser, T. Winzerling, and J. Nitsch, "Statistical interpretation of autocorrelation coefficients for fields in mode-stirred chambers," in Proc. Int. Symp. Electromagn. Compat., Chicago, IL, USA, Aug. 12, 2005, pp. 550-555.

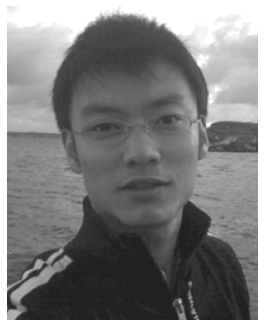

Xiaoming Chen received the B.Sc. degree in electrical engineering from Northwestern Polytechnical University, Xi'an, China, in 2006, and the M.Sc. and $\mathrm{Ph} . \mathrm{D}$. degrees in electrical engineering from Chalmers University of Technology, Gothenburg, Sweden, in 2007 and 2012, respectively.

Currently, he is a Postdoctoral Researcher in the Department of Signals and Systems, Chalmers University of Technology. His current research areas include reverberation chamber measurements, multiantenna channel characterization, over-the-air tests, and statistical electromagnetics. He is a reviewer of IEEE TRANSACTIONS ON ANTENNAS AND PRopagation, IEEE TRANSACTIONS ON ELECTROMAGNETIC COMPATIBILITY, IEEE TRANSACTIONS ON WIRELESS COMMUNICATIONS, and IEEE COMMUNICATIONS LETTERS. 